$\xi^{2}=-$

\title{
Design and Implementation of an IoT Ready Smart Sensor for Speed Sensing of a DC Motor using IEEE 802.15.1 and ESP8266
}

\author{
Ankur Jain ${ }^{1 *}$, B. K. Roy ${ }^{2}$ \\ ${ }^{1,2}$ National Institute of Technology, Silchar, India \\ *Corresponding Author Email: ' ankurjainjob@gmail.com
}

\begin{abstract}
An IoT enabled smart sensor for speed sensing of a DC motor is designed and implemented. This is a generic platform enabled with Wi$\mathrm{Fi}$, Bluetooth, etc. It can also be used for many other applications. In this paper, we have used cyber-physical aspects for the co-design of hardware and software. Our design is equipped with Bluetooth radio frequency link and TCP/IP stack. The sensor node is able to communicate to both near and far range according to the requirement. IP addresses make it uniquely identifiable. Implementation is done as per the layered architecture which makes it interoperable. A demo setup is made for verification.
\end{abstract}

Keywords: Networked control system (NCS), Internet of things (IoT), Sensor design, Geared DC motor, ESP8266.

\section{Introduction}

In the present scenario, Internet of things (IoT) is the most buzzing word in the research community. IoT may be defined as the ubiquitous scalable network of heterogeneous uniquely identifiable intelligent objects which can interact interoperability[Error!

Reference source not found.]. Acquisition of physical data, processing, and communication abilities enable IoT nodes to interact with each other through the internet or other communication networks. These nodes are constrained by their processing capability, bandwidth limitation, power, etc. On the other hand, they need to cater different quality of services for the delay, feedback, and network traffic requirement of a particular application. The present world is blessed with different wireless technologies like 3G, ZigBee, Wi-Fi, Bluetooth, etc. which give rise to ubiquitous sensing. A comparative study of the wireless network is shown in Table 1.

Table 1: A comparative study of some popular network technologies

\begin{tabular}{lcccc}
\hline Technology & $\begin{array}{c}\text { IEEE } \\
\text { standard }\end{array}$ & $\begin{array}{c}\text { Range } \\
\text { covered } \\
\text { (Meter) }\end{array}$ & $\begin{array}{c}\text { Maximum } \\
\text { data rate } \\
\text { (Mbps) }\end{array}$ & $\begin{array}{c}\text { Channel } \\
\text { bandwidth } \\
\text { (MHz) }\end{array}$ \\
\hline WiMax & 802.16 & $1-5 \mathrm{~K}$ & $30-50$ & $1.25-20$ \\
Wi-Fi & 802.11 & 100 & 54 & 22 \\
UMTS & CDMA & $0.1-10 \mathrm{~K}$ & $0.384-2$ & 5 \\
& TDMA & & & \\
ZigBee & 802.15 .4 & $10-100$ & .250 & $0.3 / 0.6 / 2$ \\
Bluetooth & 802.15 .1 & $10-100$ & 1 & 1 \\
\hline
\end{tabular}

These technologies have converted the static internet into a ubiquitous networked system[Error! Reference source not found.]. For storing these data heaps, we also have various cloud platforms on which the data can be analysed and shared among various smart objects[Error! Reference source not found., Error! Reference source not found., Error! Reference source not found., Error! Reference source not found.]. To uniquely identify a small node over an IP network and to provide connectivity over the internet with low power requirement, the 6LowPAN protocol is being used in various research areas [Error! Reference source not found.]. There are lots of domestic and civil applications where these architectures are being used such as a smart home, smart building, smart energy meters [Error! Reference source not found.], smart cities [Error! Reference source not found.], smart parking, smart garbage management [Error! Reference source not found., Error! Reference source not found., Error! Reference source not found., Error! Reference source not found., Error! Reference source not found., Error! Reference source not found.], etc. On the other hand, Industry 4.0 [Error! Reference source not found.] is also in the evolution phase where smart grid [Error! Reference source not found., Error! Reference source not found.], factory automation, smart manufacturing[Error! Reference source not found.], water quality monitoring[Error! Reference source not found., Error! Reference source not found., Error! Reference source not found., Error! Reference source not found.] are being investigated.

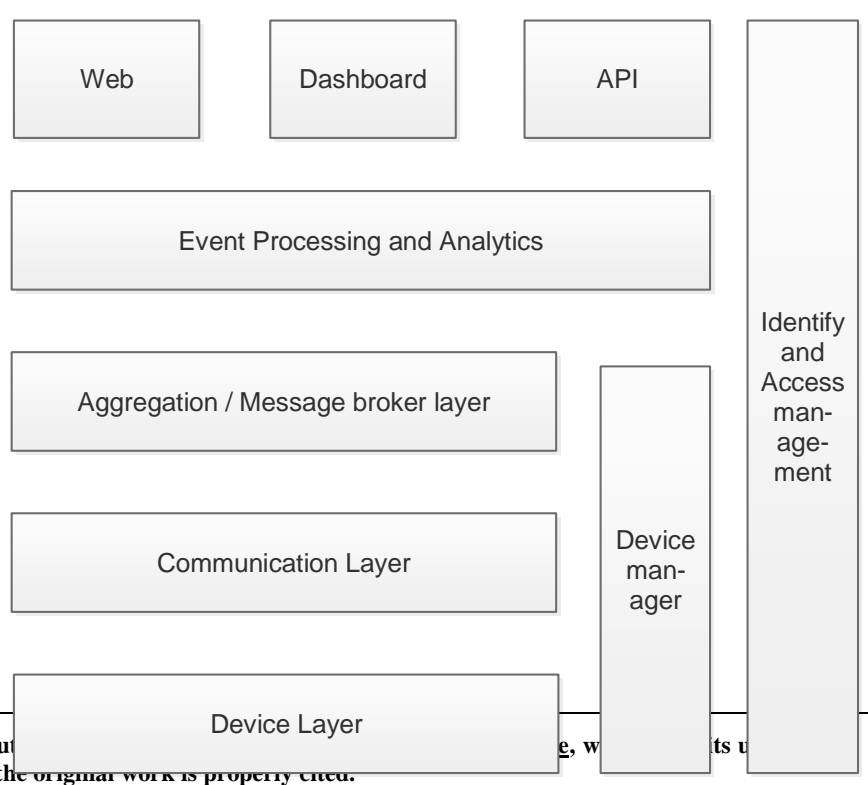


Fig. 1: Reference architecture for an IoT [Error! Reference source not found.]

IoT is a paradigm shift in the machine to machine (M2M) and machine to human interaction.

A layered reference architecture[Error! Reference source not found.]for IoT is shown in Fig. 1. Some recent applications are worth mentioning. Reference [Error! Reference source not found.]designed a smart sensor which identifies pot material of domestic cooking items. Here inductor is used as a sensing element and spectral method is used for the calculation of impedance using voltage and current waveform across sensing element. A feature set of impedance and power factor is used for learning of pot material. In [Error! Reference source not found.], smart recognition and counting of objects using a camera as a sensing element was designed. Reference [Error! Reference source not found.] explored an energy efficient model in IoT environment and proposed an architecture for integration of wireless sensor networks with IoT. Reference [Error! Reference source not found.]designed a microcontroller-based sensor for measuring water quality in real-time using ZigBee communication protocol Reference [Error! Reference source not found.]presented a wireless sensor network (WSN) based on ISO/IEC/IEEE 21451 for monitoring water bodies to achieve the objective of capturing extreme events. In [Error! Reference source not found.], authors proposes a reconfigurable design using complex programmable logic devices (CPLD). They adopted IEEE1451.2 intelligent sensor interface specifications and co-designed hardware and software interface Apart from these, some works have been reported in environment monitoring [Error! Reference source not found., Error! Reference source not found.]. The above literature review reveal that wireless sensor network standards have been established well. They have been applied in many fields. However, lot more can be done in industrial aspects like control, maintenance, real-time decision making, etc.

In this paper, we have mainly concerned with the design of a smart sensor for speed sensing of a DC motor. This design is developed for an end to end solution keeping scalability, interoperability, and extensibility in mind. Our design is capable of processing and transmitting data in a local network as well as in an internetwork scenario. Our proposed design is reconfigurable, versatile, cost effective and modular, and can be used for different other applications. With this design, sensing element could be replaced periodically without disturbing the entire system.

The paper is organised as follows: Section 2 covers all the design issues and architectures of different individual layers. Section 3 describes the implementation procedure of hardware, software, and communication module design. In Section 4, we provide the detailed experimental results. We have concluded the paper in Section 5

\section{System Design}

We have worked on the device layer, communication layer and Device management of the IoT architecture as shown in Fig 1. The Proposed layered architecture for our smart sensor node is shown in Fig. 2, where hardware and software co-design is implemented for the speed sensing.

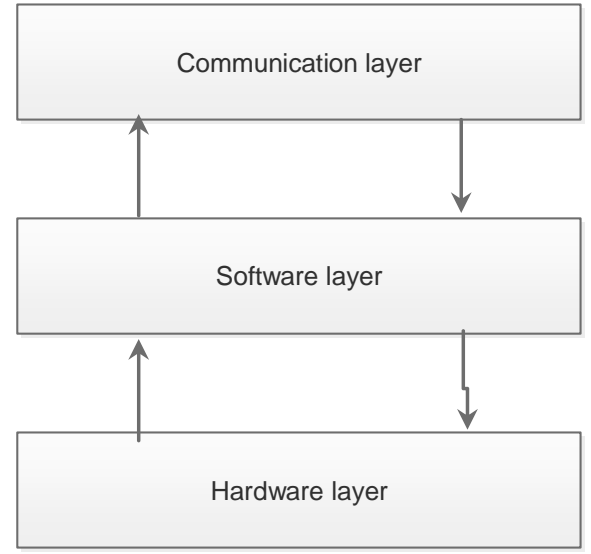

Fig. 2: Proposed layered architecture for sensor node

In a typical industrial scenario, we have to sense either analog or digital signal. A microcontroller can only process digital signals. Hence, usually, we need to convert analog data into digital data using analog to digital converter (ADC). However, in our case, we are generating only digital event to sense. Thus, there is no need of ADC. But for the sack of generalisation, we have kept the provision of an $\mathrm{ADC}$ with the hardware implementation. A schematic diagram of hardware implementation design is shown in Fig. 3.

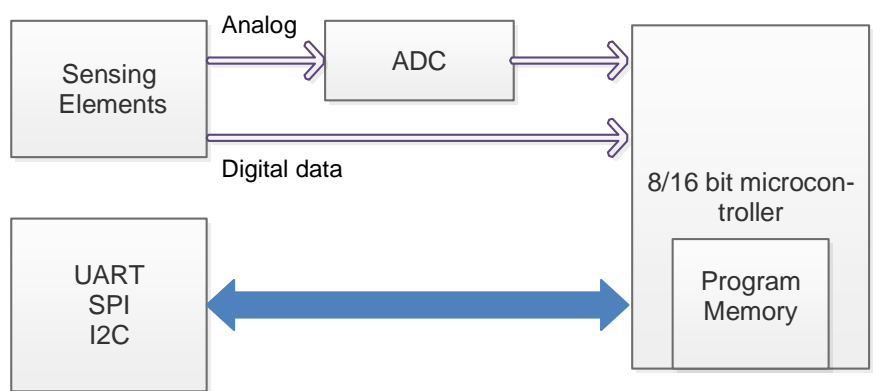

Fig. 3: Hardware design part for the sensor node

After capturing the data in digital format, we run the software algorithm to converts these bits into useful information. The software layer is attached to the hardware layer. A schematic diagram of software design is shown in Fig. 4.

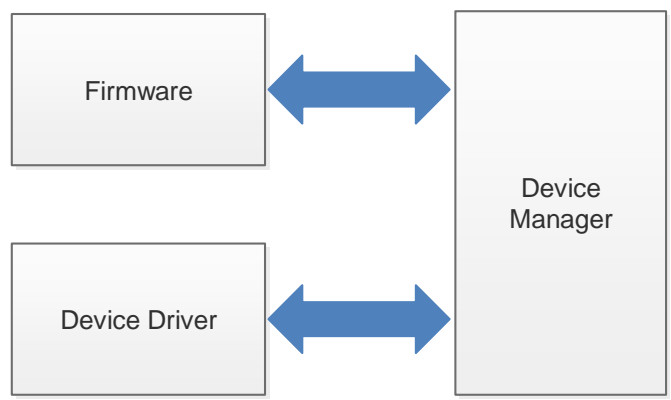

Fig. 4: Software design part for the sensor node

The information obtained from the firmware are sent to the serial port, from where according to demand requirement, the data are sent to near range, far range, or even in the cloud platform. The schematic diagram of the communication layer design is shown in Fig. 5 


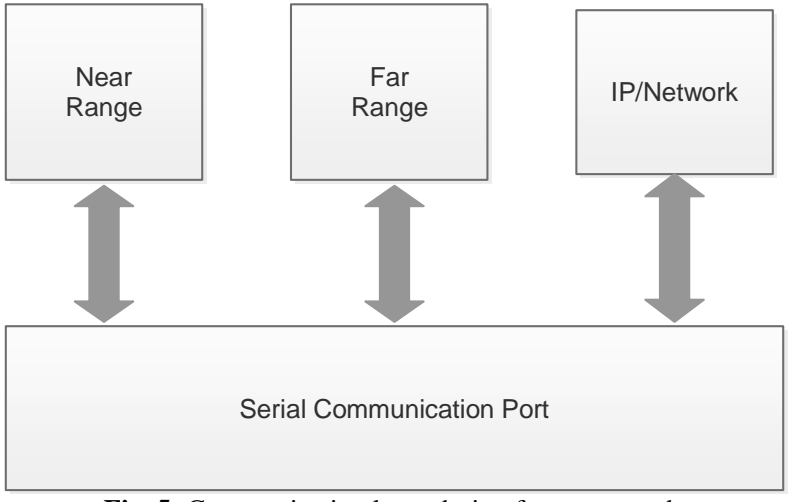

Fig. 5: Communication layer design for sensor node

The objective of the work is to create a reconfigurable interface for different sizes of the motor or other rotating elements. The design is done with a limited data processing ability for observation, estimation, and transmission. The node has a unique identification, radio frequency link, and TCP/IP stack.

\section{System Implementation}

\subsection{Hardware Layer Design}

In this design, we have used encoder disc with equally spaced 20 holes. An infrared sensor is mounted in such a way that the disc makes and break the transmission path of infrared. When IR sensor makes the path then an event is generated. ATmega2560 microcontroller is used for timing and event handling.
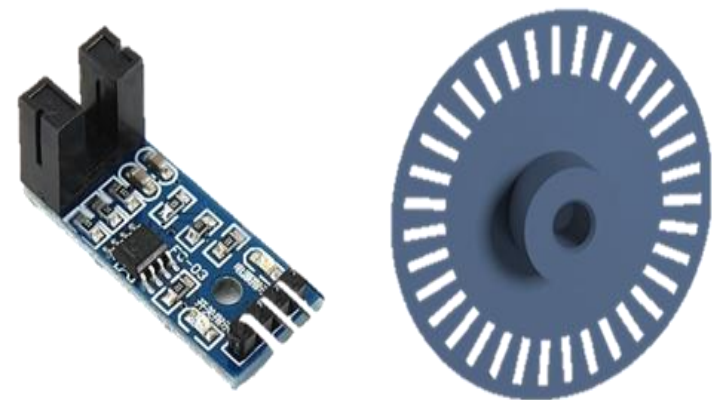

Fig. 6: (a). Infrared sensor (Left) (b). Encoder disc (Right)

\subsection{Software Layer Design}

Event monitored by the hardware is converted to the useful information, i.e. speed (in RPM) in our case, by our algorithm. An android application is designed to supervise the motor. The flowchart is shown in Fig. 7.

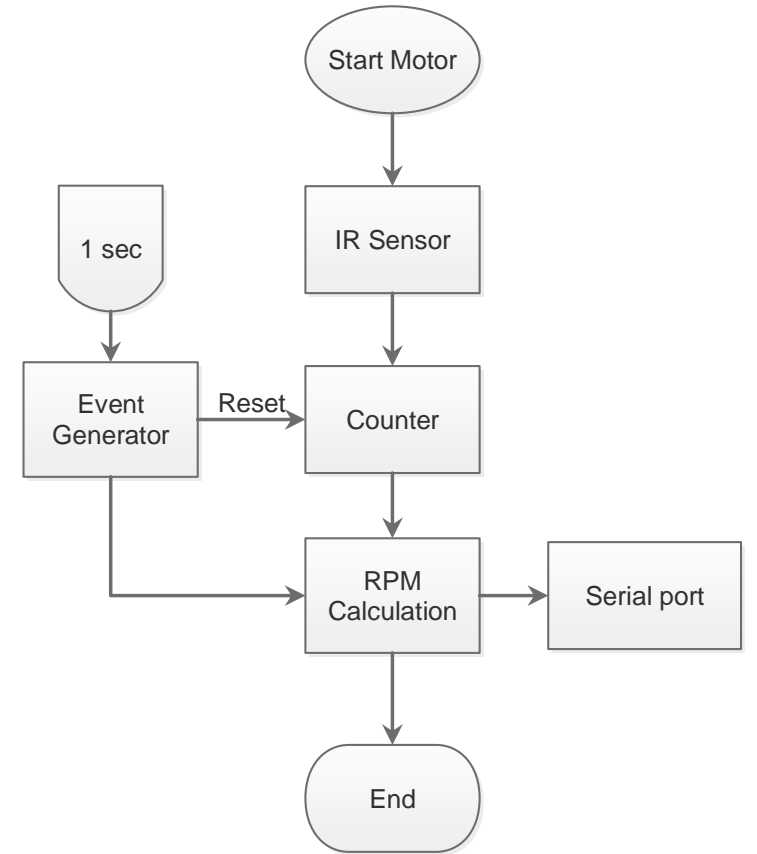

Fig. 7: Communication layer design for the sensor node

\subsection{Communication Layer Design}

We have divided this layer into three modes. The first mode provides local communication where we have used universal asynchronous receiver transmitter (UART) protocol. The second mode is used for near range communication where we have used Bluetooth protocol. With this mode, we can visualise and supervise the plant where this smart platform is mounted. For the far range communication, we have used Wi-Fi connectivity using esp8266 and for IP communication, we need TCP/IP stack.
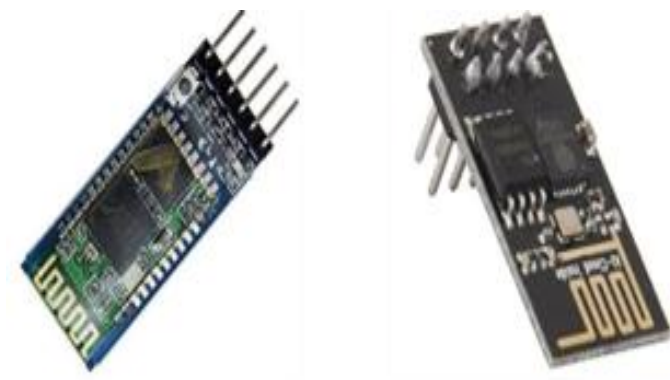

Fig. 7: (a). Bluetooth module (b). Wi-Fi module

\section{Experimental setup and Results}

We have conducted the experiment to measure the revolution per minute online over the network. The assembly used for this experiment can be seen in Fig 8. The cyber part of the experimental setup is shown in Fig. 9. Due to the wireless network, the responsiveness of the sensor may vary at different locations which depend on the distance between the plant and mobile location. 


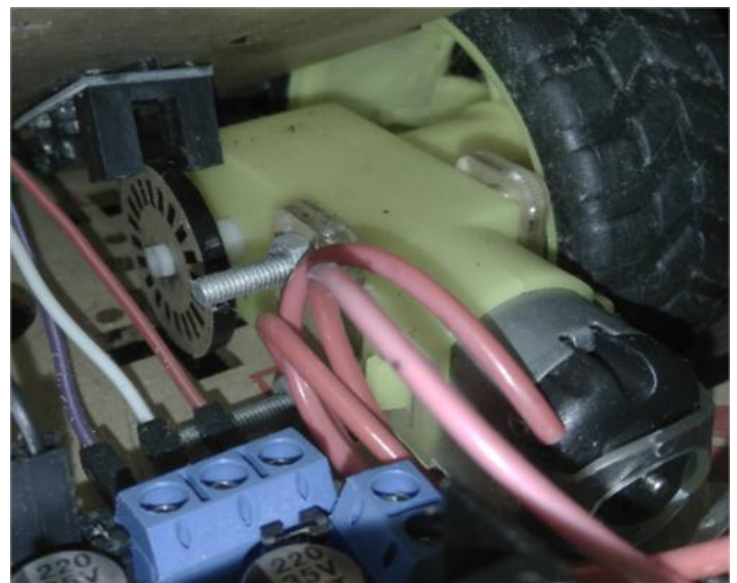

Fig. 8: Experimental smart sensor assembly setup for speed measurement

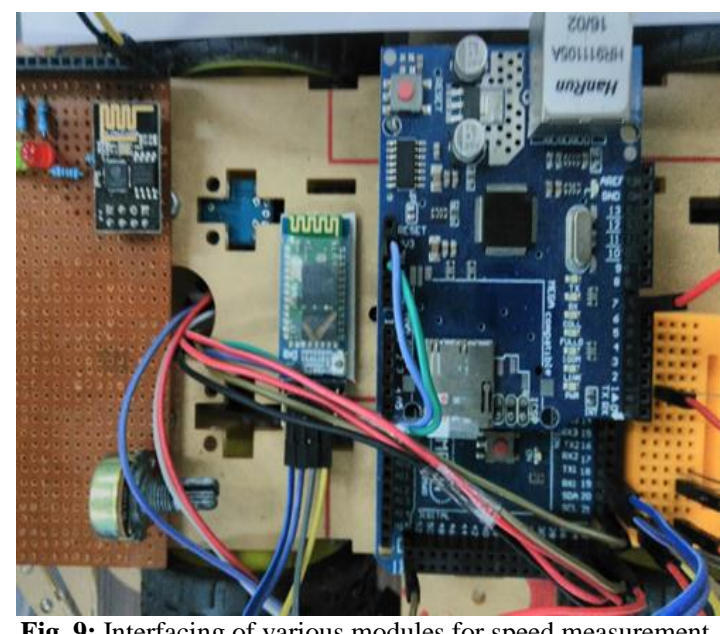

Fig. 9: Interfacing of various modules for speed measurement

An android application is used for sending the reference command to run the motor at different speeds and receiving the value of revolution per minute of the motor. It is displayed locally on a mobile and web interface, which can be seen in Fig 9. The verification of the accuracy of the proposed smart sensor is done using a tachometer (dot-791) to measure the speed locally. The results are given in Table 2 . The speed reading by the proposed smart sensor is closely matching with the reading of the tachometer. Thus, the proposed smart sensor is working satisfactorily within the experimental limitation.

Table 2: Observation table for determinationaccuracy of the sensor

\begin{tabular}{lcccc}
\hline $\begin{array}{l}\text { Voltage } \\
\text { applied }(\mathbf{V})\end{array}$ & $\begin{array}{c}\text { Duty } \\
\text { cycle } \\
(\%)\end{array}$ & $\begin{array}{c}\text { RPM }^{*} \\
\text { determined } \\
\text { (Sensor) }\end{array}$ & $\begin{array}{c}\text { RPM measured }^{*} \text { (Tachometer) } \\
\text { (in \%) }\end{array}$ & $\begin{array}{c}\text { Error } \\
\text { (Sensor }\end{array}$ \\
\hline 2.4 & 20 & 0 & 0 & 0 \\
4.8 & 40 & $\sim 238$ & 241 & 1.2 \\
7.2 & 60 & $\sim 304$ & 310 & 1.9 \\
9.5 & 80 & $\sim 333$ & 338 & 1.4 \\
12 & 100 & $\sim 356$ & 365 & 2.4 \\
\hline
\end{tabular}

*Revolution per minute, approximately

\section{Conclusions}

In this paper, we have presented the design and experimental setup of an IoT-based smart sensor for measurement of the speed of a DC motor. The proposed sensor node has communication feature which enables it to transmit measurement data successfully. The accuracy of the proposed smart sensor is compared with the measurement of a tachometer. The experimental results reveal that the proposed smart sensor is able to measure the speed correctly with- in the experimental limitation. In future, we will extend our design for cloud interfacing.

\section{Acknowledgement}

The authors acknowledge the financial support given by TEQIPIII, NIT Silchar, Silchar - 788010, Assam, India.

\section{References}

[1] Ademola P. Abidoye and Ibidun C. Obagbuwa. Models for integrating wireless sensor networks into the Internet of Things, smart sensor. IET Wireless Sensor Systems, 7(3):65-72, 2017.

[2] Francesco Adamo, Filippo Attivissimo, Carlo Guarnieri Calò Carducci, Anna Maria Lucia Lanzolla, Carlo Guarnieri Calo Carducci, and Anna Maria Lucia Lanzolla. A smart sensor network for sea water quality monitoring. IEEE Sensors Journal, 15(5):2514-2522, 2015.

[3] Gregorio Andria, Giuseppe Cavone, Vincenzo Di Lecce, and Anna Maria Lucia Lanzolla. Model characterization in measurements of environmental pollutants via data correlation of sensor outputs. IEEE transactions on instrumentation and measurement, 54(3):1061-1066, 2005.

[4] Gregorio Andria, Giuseppe Cavone, and Anna ML Lanzolla. Modelling study for assessment and forecasting variation of urban air pollution. Measurement, 41(3):222-229, 2008.

[5] Adnan Anwar, Abdun Mahmood, and Mark Pickering. Estimation of smart grid topology using SCADA measurements. 2016 IEEE International Conference on Smart Grid Communications, SmartGridComm 2016, (1):539-544, 2016.

[6] Leonardo Ascorti, Stefano Savazzi, Gloria Soatti, Monica Nicoli, Emiliano Sisinni, and Stefano Galimberti. A Wireless Cloud Network Platform for Industrial Process Automation: Critical Data Publishing and Distributed Sensing. IEEE Transactions on Instrumentation and Measurement, 66(4):592-603, 2017.

[7] Antonio Bono Nuez, Bonifacio Martin-Del-Brio, Carlos BernalRuiz, Francisco Jose Perez-Cebolla, Abelardo Martinez-Iturbe, and Ivan Sanz-Gorrachategui. The Inductor as a Smart Sensor for Material Identification in Domestic Induction Cooking. IEEE Sensors Journal, 18(6):2462-2470, 2018.

[8] Alessio Botta, Walter De Donato, Valerio Persico, and Antonio Pescapé. Integration of Cloud computing and Internet of Things: A survey. Future Generation Computer Systems, 56:684-700, 2016.

[9] Prof Sandeep M Chaware, Shriram Dighe, Akshay Joshi, Namrata Bajare, and Rohini Korke. Smart Garbage Monitoring System using Internet of Things ( IoT ).International Journal of Innovative Research in Electrical, Electronics, Instrumentation and Control Engineering 5(1):74-77, 2017.

[10] Qingping Chi, Hairong Yan, Chuan Zhang, Zhibo Pang, and Li Da $\mathrm{Xu}$. A reconfigurable smart sensor interface for industrial WSN in IoT environment. IEEE Transactions on Industrial Informatics, 10(2):1417-1425, 2014.

[11] Mehmet Hazar Cintuglu, Osama A. Mohammed, Kemal Akkaya, and A. Selcuk Uluagac. A Survey on Smart Grid Cyber-Physical System Testbeds. IEEE Communications Surveys and Tutorials, 19(1):446-464, 2017.

[12] Niel Andre Cloete, Reza Malekian, and Lakshmi Nair. Design of Smart Sensors for Real-Time Water Quality Monitoring, smart sensor. IEEE Access, 4(9):3975-3990, 2016.

[13] Manuel Diaz, Cristian Martin, and Bartolome Rubio. State-of-theart, challenges, and open issues in the integration of Internet of things and cloud computing. Journal of Network and Computer Applications, 67:99-117, 2016.

[14] Waleed Ejaz, Muhammad Naeem, Adnan Shahid, Alagan Anpalagan, and Minho Jo. Efficient Energy Management for Internet of Things in Smart Cities. IEEE Communications Magazine, (January):84-91, 2017.

[15] Paul Fremantle. A reference architecture for the internet of things. WSO2 White paper (2014).

[16] Dimitrios Georgakopoulos, Prem Prakash Jayaraman, Maria Fazia, Massimo Villari, and Rajiv Ranjan. Internet of Things and Edge Cloud Computing Roadmap for Manufacturing. IEEE Cloud Computing, 3(4):66-73, 2016.

[17] Jayavardhana Gubbi, Rajkumar Buyya, Slaven Marusic, and Marimuthu Palaniswami. Internet of Things (IoT): A vision, architectur- 
al elements, and future directions. Future Generation Computer Systems, 29(7):1645-1660, 2013.

[18] Jose M Gutierrez, Michael Jensen, Morten Henius, and Tahir Riaz. Smart Waste Collection System Based on Location Intelligence. Procedia - Procedia Computer Science, 61:120-127, 2015.

[19] N Sathish Kumar, B Vijayalakshmi, R Jenifer Prarthana, and A Shankar. IoT Based Smart Garbage alert system using Arduino UNO. In Region 10 Conference (TENCON), 2016

[20] Shih-hsiung Lee and Chu-sing Yang. A Real Time Object Recognition and Counting System for Smart Industrial Camera Sensor, Smart Sensor. 17(8):2516-2523, 2017.

[21] Shancang Li, Li Da Xu, and Shanshan Zhao. The internet of things: a survey. Information Systems Frontiers, 17(2):243-259, 2015.

[22] Chih-Wei Lu, Chih-Ming Hsieh, Chih-Hung Chang, and ChaoTung Yang. An improvement to data service in cloud computing with content sensitive transaction analysis and adaptation. In Computer Software and Applications Conference Workshops (COMPSACW), 2013 IEEE 37th Annual, pages 463-468. IEEE, 2013.

[23] Daniel Minoli. Building the Internet of Things with IPv6 and MIPv6: The Evolving World of M2M Communications. John Wiley \& Sons.2013.

[24] K. Mohammed Shahanas and P. Bagavathi Sivakumar. Framework for a Smart Water Management System in the Context of Smart City Initiatives in India. Procedia Computer Science, 92:142-147, 2016.

[25] Anitha A. Garbage monitoring system using IoT. InIOP Conference Series: Materials Science and Engineering 2017 Nov (Vol. 263, No. 4, p. 042027). IOP Publishing.

[26] Shukla, Shashank, and Neeraj Shukla. "Smart Waste Collection System based on IoT (Internet of Things): A Survey." International Journal of Computer Applications (0975-8887) Volume (2017).

[27] Putra, Dito Adhi, and Tri Harsono. "Smart sensor device for detection of water quality as anticipation of disaster environment pollution." In Electronics Symposium (IES), 2016 International, pp. $87-$ 92. IEEE, 2016..

[28] Jiafu Wan, Shenglong Tang, Zhaogang Shu, Di Li, Shiyong Wang, Muhammad Imran, and Athanasios V. Vasilakos. Software-Defined Industrial Internet of Things in the Context of Industry 4.0. IEEE Sensors Journal, 16(20):7373-7380, 2016.

[29] Yusof, Norfadzlia Mohd, Aiman Zakwan Jidin, and Muhammad Izzat Rahim. "Smart garbage monitoring system for waste management." In MATEC Web of Conferences, vol. 97, p. 01098. EDP Sciences, 2017. 